\title{
块碎石层几何参数对其自然对流降温效应影响的 数值研究
}

\author{
张明义 ${ }^{*}$, 程国栋, 李双洋 \\ 中国科学院寒区旱区环境与工程研究所 冻土工程国家重点实验室, 兰州 730000 \\ *E-mail: myzhang@lzb.ac.cn \\ 收稿日期: 2008-03-05; 接受日期: 2008-12-02 \\ 国家自然科学基金(批准号: 40601023, 40730736)和中国科学院“院长奖获得者科研启动专项资金”资助项目
}

\begin{abstract}
摘要块碎石层作为一种高渗透率的多孔介质, 经常应用于寒区道路的护坡工程中, 用于保护 其下部多年冻土, 提高道路的稳定性. 本文为提高和优化寒区道路工程中块碎石护坡的降温能 力, 基于多孔介质中流体热对流理论, 根据野外块碎石护坡的实际几何尺寸及热边界条件等因 素, 采用稳态模型对厚度为 $1.0 \mathrm{~m}$, 底顶温差为 $10^{\circ} \mathrm{C}$, 不同倾斜角度、不同长厚比等几何条件的块 碎石层中对流模式及降温效果进行了数值研究. 结果表明: 在本计算条件下, 无论在何种倾斜角 度及长厚比条件下, 由于块碎石层内部空气自然对流的存在均可提高其等效导热系数, 使其具有 热半导体特性, 但内部的对流模式会随着倾斜角度的变化而发生改变, 对流环数随着其倾斜角度 的增大而减少, 当对流环由多个转变为单个时, 对应的 $N u$ 数最小, 降温效果最差, 此时对应的倾 斜角度为块碎石层最差降温倾斜角度. 并且, 该最差降温倾斜角度随着其长厚比的增大而增大, 最终大致趋近于 $32^{\circ}$, 与野外一般块碎石护坡的倾斜角度 $33.7^{\circ}$ 十分接近. 因此, 当野外块碎石护 坡路基高度较大, 即块碎石护坡长厚比过大时, 应采取必要措施来提高块碎石护坡的降温能力, 本文在这方面也进行了必要的研究和讨论, 以期为寒区块碎石护坡路基的设计与维护提供一些 科学参考.
\end{abstract}

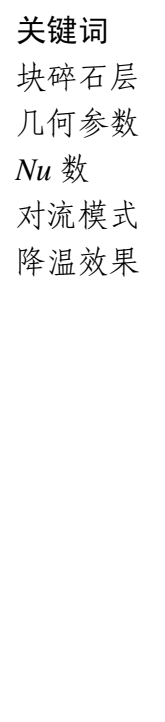

\section{1 引言}

由于块碎石层可以看成是具有高渗透率的多孔 介质, 在满足一定条件时内部会有空气自然对流发 生 ${ }^{[1,2]}$, 正是其具有多孔介质的这种自然对流降温效 应, 在寒区道路工程建设中为了保护下部多年冻土, 确保道路的安全稳定, 得到了广泛的应用 ${ }^{[3 \sim 9]}$. 块碎 石护坡是寒区道路工程中的一种主要路基结构形式, 人们对其降温效果已经进行了大量的研究. 例如,
张明义等 ${ }^{[10]}$ 通过室内试验研究发现粒径对块碎石护 坡降温效果有明显的影响; 孙志忠等 ${ }^{[6]}$ 通过青藏铁路 野外试验发现块石与碎石护坡均对其下部多年冻土 具有积极的保护作用; 赖远明等 ${ }^{[11]}$ 基于多孔介质对 流传热理论, 对青藏铁路块碎石护坡路基降温效果 进行了数值计算分析, 发现通过调整块碎石护坡的 厚度可解决冻土路基阴阳坡问题, 减少道路病害的 发生. 这些研究表明块碎石护坡对下部多年冻土具 有很好的保护降温作用, 可有效确保寒区道路的多

引用格式: Zhang M Y, Cheng G D, Li S Y. Numerical study on the influence of geometrical parameters on natural convection cooling effect of the crushed-rock revetment. Sci China Tech Sci, 2009, 52(2): 539-545, doi: 10.1007/s11431-008-0329-9 
年稳定.

目前, 有研究表明 ${ }^{[12 \sim 14]}$ : 多孔介质层的长宽比、 倾斜角度等参数的变化会引起其对流模式、传热特性 等的变化, 从而对其降温效果产生显著影响. 但以往 对块碎石护坡的研究中主要考虑了粒径、厚度等参数 对其降温效果的影响, 一些其他参数, 例如长厚比、 倾斜角度等对块碎石护坡降温效果的影响目前未见 报道. 由于这些几何参数会随着路基高度、坡角等发 生变化, 从而对其对流模式及降温效果产生影响, 所 以对这些参数的研究也是非常必要, 并且具有实际 工程意义. 因此, 本文根据多孔介质中流体热对流的 连续性方程、动量方程和能量方程, 建立了用于计算 块碎石护坡流场和温度场的数值计算模型, 对不同 倾斜角度、不同长厚比的块碎石层的对流模式和传热 特性进行了数值研究, 以期为寒区块碎石护坡路基 的设计与维护提供参考.

\section{2 理论与数值模型}

块碎石护坡可以看成是多孔介质区, 为了简化 计算, 做如下基本假设:

1) 气体不可压缩，符合 Boussinesq 假设;

2) 多孔介质与其内部气体处于局部热平衡状态;

3) 由于块碎石护坡沿路基长度方向可看作无限 长, 因此简化为横断面方向的二维各向同性问题.

基于以上假设, 计算模型采用二维矩形, 见图 1 . 研究中, 定义边界 $\mathrm{AB}$ 和 $\mathrm{CD}$ 为长度, $\mathrm{AD}$ 和 $\mathrm{BC}$ 为厚度, 长度和厚度的比值为长厚比, $\varphi$ 是倾斜角度; 设上下表 面温度恒定, 取上表面 $\mathrm{AB}$ 温度 $T_{\mathrm{AB}}$ 低于下表面 $\mathrm{CD}$ 温 度 $T_{\mathrm{CD}}$, 边界 $\mathrm{BC}$ 和 $\mathrm{AD}$ 设为绝热边界. 其对流换热控制 方程组为连续性方程、动量方程和能量方程 ${ }^{[15 ~ 17]}$ :

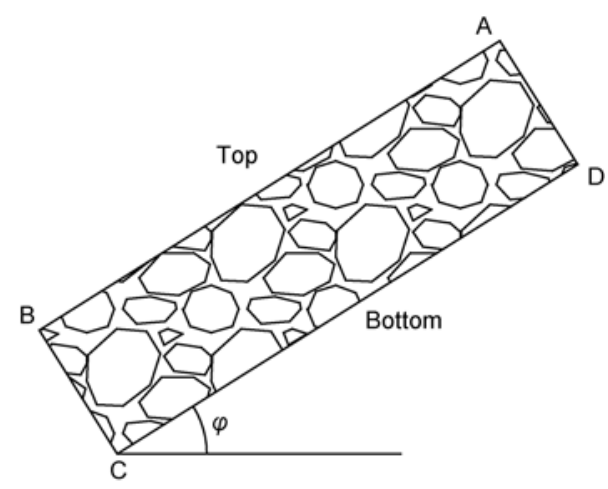

图 1 块碎石层横断面
连续性方程:

$$
\frac{\partial v_{x}}{\partial x}+\frac{\partial v_{y}}{\partial y}=0
$$

式中, $v_{x}, v_{y}$ 分别为空气在 $x$ 和 $y$ 方向上的渗流速度分量. 动量方程:

$$
\begin{gathered}
\frac{\partial p}{\partial x}=-\frac{\mu}{k} v_{x}-\rho B|v| v_{x}, \\
\frac{\partial p}{\partial y}=-\frac{\mu}{k} v_{y}-\rho B|v| v_{y}-\rho_{\alpha} g,
\end{gathered}
$$

式中, $|v|=\left(v_{x}^{2}+v_{y}^{2}\right)^{1 / 2}, B$ 为惯性阻力系数, $k$ 为多孔 介质的渗透率, $\mu$ 为空气的动力粘度, $p$ 为空气压力, $\rho B|v| v_{x}$ 为惯性损失项.

考虑空气是不可压缩的, 但其密度 $\rho_{a}$ 是温度的 函数, 为了简化分析, 使用 Boussinesq 假设. 即: 只 有重力项中的空气密度是可变的, 可表示为:

$$
\rho_{\alpha}=\rho_{0}\left[1-\beta\left(T-T_{0}\right)\right],
$$

式中, $\beta$ 为空气的热膨胀系数, $\rho_{0}$ 和 $T_{0}$ 分别为空气密 度和温度的参考值.

能量方程:

$$
\frac{\partial\left(v_{x} T\right)}{\partial x}+\frac{\partial\left(v_{y} T\right)}{\partial y}=\alpha\left(\frac{\partial^{2} T}{\partial x^{2}}+\frac{\partial^{2} T}{\partial y^{2}}\right),
$$

式中, $\alpha=\frac{\phi \lambda_{a}+(1-\phi) \lambda_{s}}{\rho c_{p}}$, 其中 $c_{p}$ 为空气的定压比热, $\phi$ 是块碎石层孔隙率, $\lambda_{a}$ 和 $\lambda_{s}$ 分别是多孔介质层气体 和固体部分导热系数.

对于这种特殊封闭条件, 为了计算方便可引入 流函数 $\psi$, 则

$$
\begin{gathered}
v_{x}=\frac{\partial \psi}{\partial y}, \\
v_{y}=-\frac{\partial \psi}{\partial x} .
\end{gathered}
$$

将(5a)和(5b)分别代入(2a)和(2b), 可得:

$$
\begin{gathered}
\frac{\partial p}{\partial x}=-\frac{\mu}{k} \frac{\partial \psi}{\partial y}-\rho B|v| \frac{\partial \psi}{\partial y}, \\
\frac{\partial p}{\partial y}=\frac{\mu}{k} \frac{\partial \psi}{\partial x}+\rho B|v| \frac{\partial \psi}{\partial x}-\rho_{\alpha} g .
\end{gathered}
$$

将(6a)和(6b)式分别关于 $y$ 和 $x$ 求导相减后得到 
下式:

$$
\begin{aligned}
& \left(1+\frac{k B \rho}{\mu}|v|\right)\left(\frac{\partial^{2} \psi}{\partial x^{2}}+\frac{\partial^{2} \psi}{\partial y^{2}}\right) \\
= & -\frac{\rho B k}{\mu}\left(\frac{\partial|v|}{\partial x} \frac{\partial \psi}{\partial x}+\frac{\partial|v|}{\partial y} \frac{\partial \psi}{\partial y}\right)-\frac{\rho_{0} \beta k g}{\mu} \frac{\partial T}{\partial x} .
\end{aligned}
$$

引进无量纲量:

$$
x_{D}=\frac{x}{H}, y_{D}=\frac{y}{H}, \psi_{D}=\frac{\psi}{\alpha}, v_{D}=\frac{v H}{\alpha}, \theta=\frac{T-T_{0}}{\Delta T},(8)
$$

式中, $H$ 为块碎石厚度, $\Delta T$ 为块碎石层下表面和上表 面温差.

则(7)和(4)式变为:

$$
\begin{aligned}
& \left(1+B e\left|v_{D}\right|\right)\left(\frac{\partial^{2} \psi_{D}}{\partial x_{D}{ }^{2}}+\frac{\partial^{2} \psi_{D}}{\partial y_{D}{ }^{2}}\right) \\
& =-B e\left(\frac{\partial\left|v_{D}\right|}{\partial x_{D}} \frac{\partial \psi_{D}}{\partial x_{D}}+\frac{\partial\left|v_{D}\right|}{\partial y_{D}} \frac{\partial \psi_{D}}{\partial y_{D}}\right)-R a \frac{\partial \theta}{\partial x_{D}}, \\
& \frac{\partial \psi_{D}}{\partial y_{D}} \frac{\partial \theta}{\partial x_{D}}-\frac{\partial \psi_{D}}{\partial x_{D}} \frac{\partial \theta}{\partial y_{D}}=\frac{\partial^{2} \theta}{\partial x_{D}^{2}}+\frac{\partial^{2} \theta}{\partial y_{D}^{2}}
\end{aligned}
$$

式中, $\left|v_{D}\right|=\left(\left(\frac{\partial \psi_{D}}{\partial x_{D}}\right)^{2}+\left(\frac{\partial \psi_{D}}{\partial y_{D}}\right)^{2}\right)^{1 / 2}, B e=\frac{\alpha B k \rho}{\mu H}, R a=$ $\frac{k g \beta \rho_{0} H \Delta T}{\mu \alpha}$.

边界条件设定如下:

$$
\begin{gathered}
\psi_{D}=0, \theta=-0.5, \text { 在 } \mathrm{AB} \text { 边, } \\
\psi_{D}=0, \theta=0.5, \text { 在 } \mathrm{CD} \text { 边, } \\
\psi_{D}=0, \frac{\partial \theta}{\partial n}=0, \text { 在 } \mathrm{AD} \text { 和 } \mathrm{BC} \text { 边. }
\end{gathered}
$$

由于求解以上方程组是一个强非线性问题, 无 法求得解析解, 故计算中采用数值计算方法: 首先利 用有限体积法 ${ }^{[18 \sim 20]}$ 对以上控制方程组进行离散, 然 后结合相应的边界条件, 采用逐次亚松弛迭代法对这 些离散的耦合代数方程进行求解，收敛判别条件为:

$$
\sum_{i, j}\left|\Phi_{i, j}^{N}-\Phi_{i, j}^{N-1}\right| / \sum_{i, j}\left|\Phi_{i, j}^{N}\right| \leq 10^{-6},
$$

式中, $\Phi$ 代表计算中的各变量, $N$ 为迭代步数.

\section{3 计算结果和讨论}

根据块碎石护坡的相关资料 ${ }^{[5,6,11,21]}$, 研究中采
用块碎石护坡垂直厚度 $(\mathrm{AD}$ 和 $\mathrm{BC}$ ) 为 $1.0 \mathrm{~m}$, 块碎石 平均粒径 $10 \mathrm{~cm}$, 等效导热系数 $\lambda=\phi \lambda_{a}+(1-\phi) \lambda_{s}=$ $0.387 \mathrm{~W} \cdot \mathrm{m}^{-1} \cdot{ }^{\circ} \mathrm{C}^{-1}$, 渗透率 $k=1.58 \times 10^{-6} \mathrm{~m}^{2}$, 惯性阻力 系数 $B=840.32 \mathrm{~m}^{-1}$; 取上下边界 $(\mathrm{CD}$ 和 $\mathrm{AB}$ )温差 $\Delta T=$ $T_{\mathrm{CD}}-T_{\mathrm{AB}}$ 为 $10.0^{\circ} \mathrm{C}$, 由(11a)和 $(11 \mathrm{~b})$ 可知, 上边界 $\mathrm{AB}$ 为定温 $-5.0^{\circ} \mathrm{C}$, 下边界 $\mathrm{CD}$ 为定温 $5.0^{\circ} \mathrm{C}$.

同时, 取空气在常压条件下的参数为: 定压比热 $c_{p}=1.004 \mathrm{~kJ} \cdot(\mathrm{kg})^{-1} \cdot{ }^{\circ} \mathrm{C}$, 导热系数 $\lambda_{a}=0.02 \mathrm{~W} \cdot \mathrm{m}^{-1} \cdot{ }^{\circ} \mathrm{C}$, 密 度 $\rho=1.20 \mathrm{~kg} \cdot \mathrm{m}^{-3}$, 动力粘度 $\mu=1.75 \times 10^{-5} \mathrm{~kg} \cdot \mathrm{m}^{-1} \cdot \mathrm{s}$.

以下对厚度为 $1.0 \mathrm{~m}$, 长厚比为 $0.5: 1,1: 1,2: 1$, $3: 1,4: 1,5: 1,6: 1,7: 1$ 和 8:1 九种情况下的块碎石层, 在不同倾斜角度 $\varphi$ 条件下的对流模式和传热特性进 行数值研究.

为了便于分析和比较, 引入 $N u$ 数来判断块碎石 多孔介质层自然对流降温效果, 其表达式为:

$$
N u=\frac{\lambda^{*}}{\lambda}=\frac{q H}{\Delta T \lambda},
$$

式中, $\lambda=\phi \lambda_{a}+(1-\phi) \lambda_{s}$, 为块碎石层的固有等效导 热系数(无对流条件), $\lambda^{*}$ 为块碎石层的实际等效导热 系数(有或无对流条件). 我们可以通过 $N u$ 数的大小 直接判断出块碎石层降温效果的变化.

图 2 为 9 种长厚比块碎石层的 $N u$ 数随其倾斜角 度变化情况. 从图 2 中可以看出, 在这 9 种长厚比的 情况下, 当块碎石层上表面(AB)温度低于下表面(CD) 时, 无论倾斜角度多大, 其 $N u$ 数均大于 1.0 , 这表明 块碎石层中空气对流是始终存在, 并且这种空气自 然对流增加了其等效导热系数. 另外, 块碎石层在不 同长厚比的情况下, $N u$ 数随其倾斜角度的变化规律 总体是相同的：只有当长厚比为小于或等于 $1: 1$ 时, 倾角 $\varphi$ 为 $0^{\circ}$ 时 $N u$ 数最小, 而其他情况 $N u$ 数均表现为 先出现迅速减小, 达到最小后随着倾斜角度的增大 而缓慢增大, 随后又出现减小趋势. 这种变化规律表 明块碎石层的对流降温效果存在一个最差的倾斜角 度, 从图 2 大致得到了以上 9 种长厚比条件下块碎石 层最差降温倾斜角度, 列于表 1 . 为了便于分析和比 较, 将表 1 中不同长厚比与对应的最差降温倾斜角度 绘于图 3 .

从表 1 和图 3, 我们不难看出随着长厚比的增大, 碎石层最差降温倾斜角度也在不断增大, 但变化速 率逐渐减慢, 并且从计算和相关理论研究结果 ${ }^{[13,14]}$ 发现：块碎石层厚度为 $1.0 \mathrm{~m}$ 条件下, 这个最差降温 
表 1 不同长厚比条件下块碎石层最差降温倾斜角度及对应的 $\mathrm{Nu}$ 数

\begin{tabular}{cccccccccc}
\hline 长厚比 & $0.5: 1$ & $1: 1$ & $2: 1$ & $3: 1$ & $4: 1$ & $5: 1$ & $6: 1$ & $7: 1$ & $8: 1$ \\
\hline 最差降温倾斜角度 $\left({ }^{\circ}\right)$ & 0 & 0 & 9 & 23 & 26 & 27 & 28 & 29 & 30 \\
$N u$ 数 & 1.97 & 2.32 & 1.98 & 1.83 & 1.68 & 1.55 & 1.47 & 1.40 & 1.36 \\
\hline
\end{tabular}

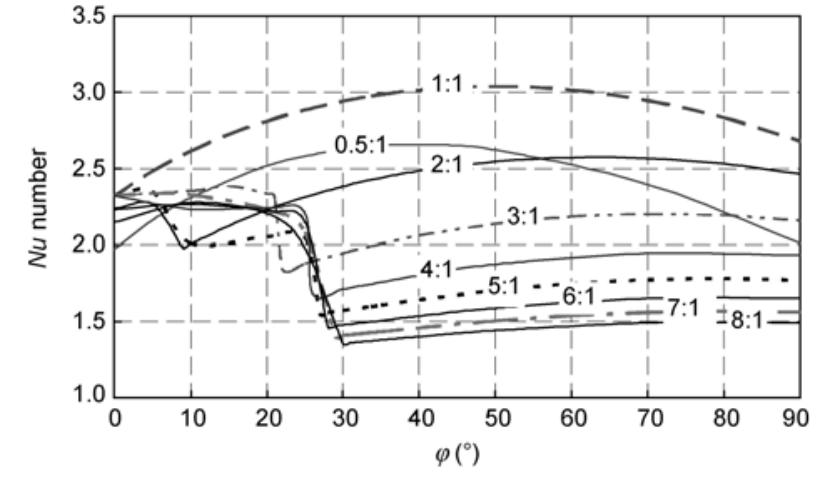

图 2 不同长厚比的块碎石层 $N u$ 数随倾斜角度的变化曲线

倾斜角度会随着长厚比的增大大致趋于 $32^{\circ}$. 目前, 在野外实体工程中护坡的坡度一般采用 $1: 1.5$, 倾斜 角度约为 $\operatorname{arctg}(1 / 1.5) \approx 33.7^{\circ}$, 与理论计算得到的 $32^{\circ}$ 十分接近, 这可能对块碎石护坡降温效果的发挥不 利. 因此, 在实际工程中块碎石护坡在封闭或无风条 件下要注意倾斜角度的选择.

我们将不同长厚比条件下最差降温倾斜角度及 $33.7^{\circ}$ 倾斜角度对应的 $N u$ 数绘于图 4. 从图 4 中, 我们 可以看到, $33.7^{\circ}$ 倾斜角度和最差降温倾斜角度对应 的 $N u$ 数均在长厚比为 $1: 1$ 时出现最大值, 并且在长 厚比较小的时候, $33.7^{\circ}$ 倾斜角度对应的 $N u$ 数明显大 于最差降温倾斜角度对应的 $N u$ 数. 我们也发现, $33.7^{\circ}$ 倾斜角度对应的 $N u$ 数在长厚比小于 $3: 1$ 的情况 下, 均大于 2 , 这对发挥块碎石护坡的降温能力是有 利的, 而在长厚比超过 $4: 1$ 的时候, 趋近于最小 $N u$ 数, 二者相差越来越小, 这对于块碎石护坡的降温能力 的发挥是不利的. 因此, 在野外块碎石护坡工程中, 当路基高度较大时，随着块碎石护坡的长厚比增大， 其降温效率必然降低. 为了提高其工作效率, 必须采 取一些工程措施, 但考虑到工程设计及施工的需要, 我们必须在不改变护坡倾斜角度的情况下来优化块 碎石护坡的结构, 来提高其降温效果. 从图 2 我们可 以看到, 在长厚比为 $0.5: 1,1: 1$ 和 2:1 三种情况下, $N u$ 数的最大值出现在最小值之后, 并且 $N u$ 数始终保持 较大, 最小值也达到 $1.97,2.32$ 和 1.98 (表 1), 结合

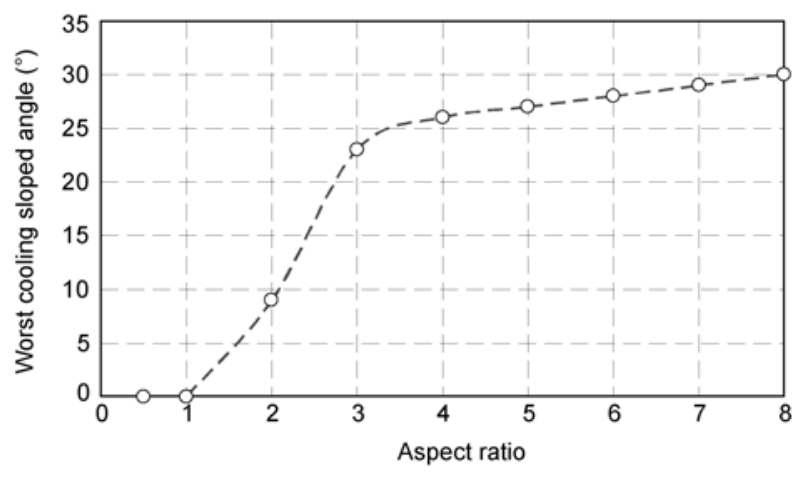

图 3 块碎石层最差降温倾斜角度随长厚比的变化曲线

图 4, 我们知道在 $0.5: 1,1: 1$ 和 2:1 三种情况下, $33.7^{\circ}$ 倾斜角度对应的 $N u$ 数分别为 $2.65,2.97$ 和 2.42 , 虽然 未达到最大，但大于 2 , 这说明块碎石护坡在倾斜角 度为 $33.7^{\circ}$ 时, 仍可将等效导热系数提高 2 倍以上, 这 对于护坡的降温能力的发挥是很有利的. 因此, 在野 外块碎石护坡长厚比过大的情况下, 可以将块碎石 护坡按一定长厚比分割成若干段来提高块碎石护坡 的降温效果, 但考虑到工程施工和工程造价问题, 建 议采用长厚比介于 $1: 1$ 和 2:1 之间, 如图 5 所示的方法.

那么块碎石层存在最差降温倾斜角度的原因是 什么? 下面我们对其温度场和对流场进行分析. 考虑 到温度场和对流场随块碎石层长厚比的变化规律基本

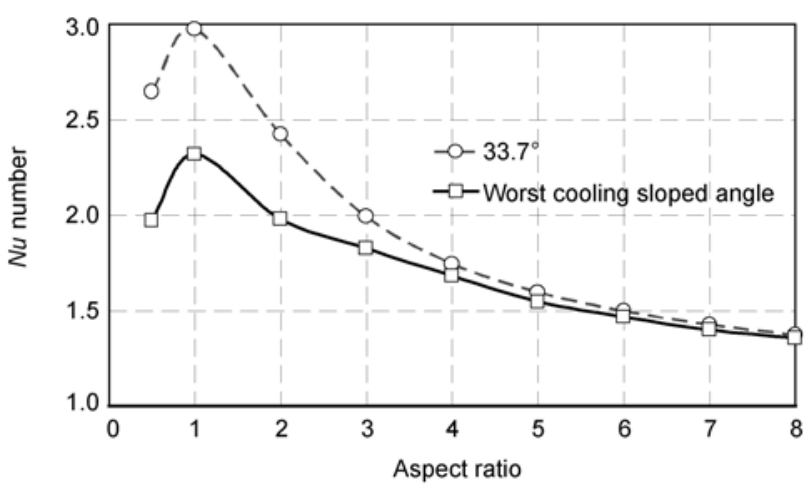

图 4 不同长厚比条件下最差降温倾斜角度与 $33.7^{\circ}$ 倾斜角度对应 $\mathrm{Nu}$ 数 


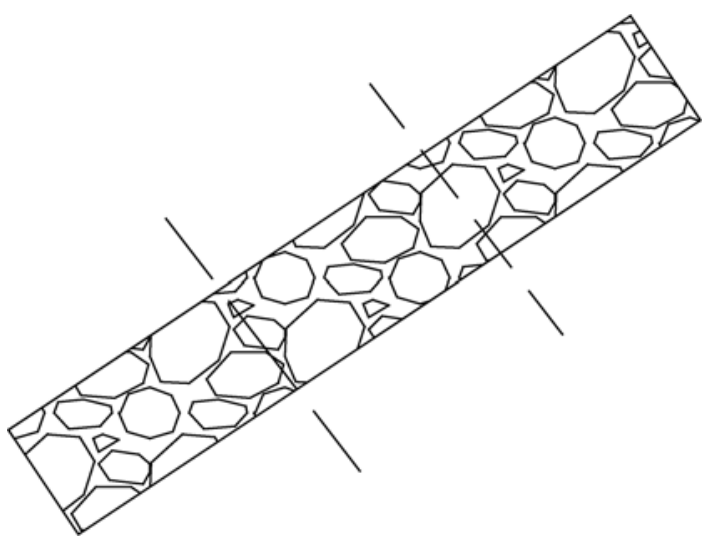

图 5 块碎石护坡按一定长厚比分割成段示意图

一致, 故选择长厚比为 7:1 的块碎石层中的温度场和 对流场变化进行分析.

图 6 显示了长厚比为 7:1, 不同倾斜角度条件下 块碎石层中的温度场与速度场的变化情况. 从图 6 中 我们可以发现块碎石层中对流环数随着倾角的增大 而减少, 由 $0^{\circ}$ 倾斜角度时的 7 个对流环发展为 $30^{\circ}$ 时 的 1 个, 这表明倾斜角度影响着块碎石层中的空气对 流模式和对流强度, 进而改变其温度分布和降温能 力. 前面的研究(图 2 和表 1)表明长厚比为 7:1 时块碎 石层的最差降温倾斜角度约为 $29^{\circ}$, 这也恰恰说明块 碎石层出现最差降温角度的原因在于对流模式的改 变, 也就是说当块碎石层中对流环由多个变为单个 时对应的倾斜角度就是最差降温倾斜角度. 因此, 这 个由多环向单环过渡区间出现 $N u$ 数急剧减小的现象, 提示我们对于块碎石在护坡工程中的应用要考虑其 倾斜角度的选择, 避开最差降温倾斜角度, 以使其发 挥更大的作用.

从以上分析来看, 块碎石护坡作为一种保护冻 土的工程措施是有效的, 在本计算条件下其无论在 任何角度及长厚比条件下, 均具有热半导体特性, 但 在何种几何参数条件下其降温效果能够达到较为理 想值得我们去关注. 另外, 本研究中采用的空气密度 为常压条件下的, 而如果将块碎石护坡应用于高原 多年冻土区的道路工程中, 例如青藏铁路、青藏公路 等, 还必须考虑空气密度问题, 高原地区较小的空气 密度将会导致空气对流减弱, 并且单位体积的空气 携带热量减少, 降温能力必然降低, 因此, 在这些特 殊条件下去修筑块碎石护坡, 其参数的选择更应引 起设计与施工部门的注意.

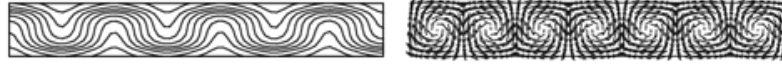

(a) $\varphi=0^{\circ}$

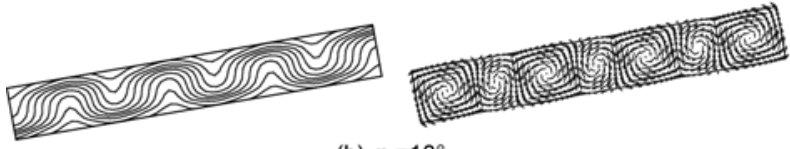

(b) $\varphi=10^{\circ}$

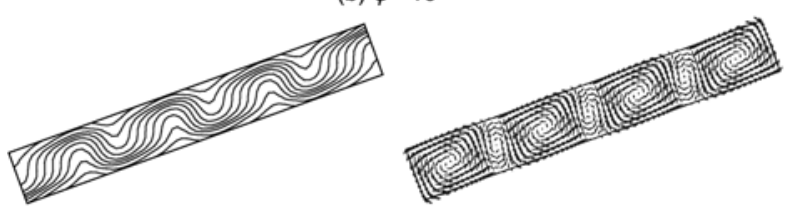

(c) $\varphi=20^{\circ}$

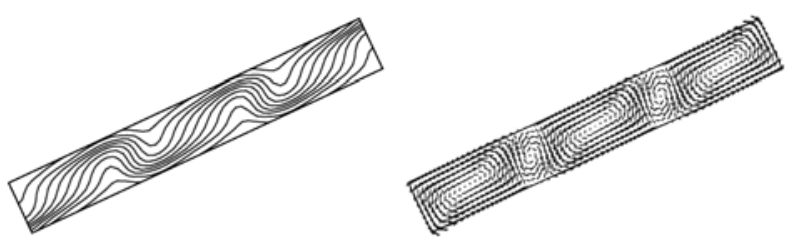

(d) $\varphi=25^{\circ}$

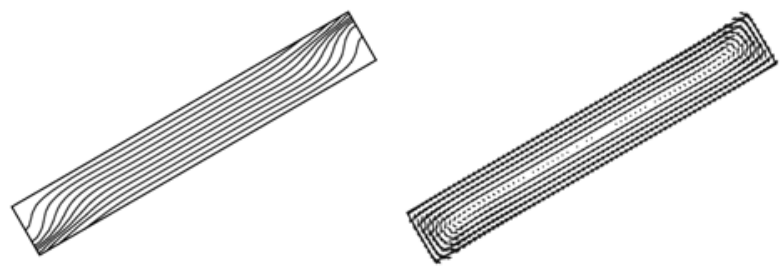

(e) $\varphi=30^{\circ}$

(B) Velocity fields

图 6 不同倾斜角度条件下块碎石层中的温度场与 速度场(长厚比为 7:1)

\section{4 结论}

本研究根据野外块碎石护坡的实际参数及相关 条件, 对厚度为 $1.0 \mathrm{~m}$ 的块碎石层在不同倾斜角度、 不同长厚比条件下的对流模式及降温效果进行了数 值研究, 得到以下初步结论:

1）在本计算条件下，无论在任何倾斜角度及长 厚比条件下，块碎石层均具有热半导体特性，但内部 的对流模式不同，对流环数随着其倾斜角度的增大而 减少, 并且当对流由多环转变为单环时, 对应的 $N u$ 数 达到最小, 也就是说此时块碎石层的降温效果最差, 对应的倾斜角度为块碎石层最差降温倾斜角度.

2) 研究中还发现, 块碎石层厚度为 $1.0 \mathrm{~m}$ 条件 下，最差降温倾斜角度随着其长厚比的增大而增大， 最终大约趋近于 $32^{\circ}$, 与野外一般块碎石护坡的倾斜 角度 $33.7^{\circ}$ 十分接近. 因此, 当长厚比过大时应采取 
必要措施来提高块碎石护坡的降温能力, 例如文中 提到的将块碎石层按一定长厚比分割成若干段, 考
虑到工程施工和工程造价问题，建议采用的长厚比 介于 $1: 1$ 和 2:1 之间.

\section{参考文献}

1 Lai Y M, Zhang L X, Zhang S J, et al. Cooling effect of ripped-stone embankments on Qinghai-Tibet railway under climatic warming. Chin Sci Bull, 2003, 48(6): 598-604

2 Zhang M Y, Lai Y M, Yu W B, et al. Laboratory investigation of the heat transfer characteristics of a trapezoidal crushed-rock layer under impermeable and permeable boundaries. Exper Heat Trans, 2006, 19(4): 251-264

3 Rooney J W. Rock fill embankment applications for convective foundation cooling on the BAM railway system. Proceeding of the Fifth International Symposium on Cold Region Development. An-chorage: ISCORD, 1997, 399-402

4 He G S, Ding J K, Li Y Q. Heat transmission properties and applica-tion of the dump filling crushed stone layer (in Chinese). J Glaciol Geocryol, 2000, 22 (Suppl): 33-37

5 吴青柏, 赵世运, 马巍, 等. 青藏铁路块石路基结构的冷却效果监测分析. 岩土工程学报, 2005, 27(12): 1386一 1390

6 Sun Z Z, Ma W, Li D Q. In situ test on cooling effectiveness of air convection embankment with crushed rock slope protection in per-mafrost regions. J Cold Reg Eng, 2005, 19(2): 38-51

7 Goering D J, Kumar P. Winter-time convection in open-graded em-bankments. Cold Reg Sci Technol, 1996, 24(1): 57-74

8 Goering D J. Passively cooled railway embankments for use in per-mafrost areas. J Cold Reg Eng, 2003, 17(3): 119-133

9 Lai Y M, Li J J, Niu F J. et al. Nonlinear thermal analysis for Qing-Tibet Railway embankments in cold regions. J Cold Reg Eng, 2003, 17(4): $171-184$

10 Zhang M Y, Lai Y M, Li S Y, et al. Laboratory investigation on cooling effect of sloped crushed-rock revetment in permafrost regions. Cold Reg Sci Technol, 2006, 46 (1): 27-35

11 Lai Y M, Zhang S J, Zhang L X, et al. Adjusting temperature distri-bution under the south and north slopes of embankment in permafrost regions by the ripped-rock revetment. Cold Reg Sci Technol, 2004, 39(1): 67-79

12 Bories S A, Combarnous M A. Natural convection in a sloping porous layer. J Fluid Mech, 1973, 57: 63-79

13 Caltagirone J P, Bories S. Solutions and stability of criteria of natural convective flow in an inclined porous layer. J Fluid Mech, 1985, 155: $267-287$

14 Cheng G D, Lai Y M, Sun Z Z, et al. The 'thermal semi-conductor' effect of crushed rocks. Permafrost and Periglacial Processes, 2007, 18: $151-160$

15 孔祥言. 高等渗流力学. 合肥: 中国科学技术大学出版社, 1999

16 Nield D A, Bejan A. Convection in Porous Media. 2nd ed. New York: Springer-Verlag, 1999

17 孔祥言, 吴建兵. 多孔介质中的非达西自然对流的分盆研究. 力学学报, 2002, 34(2): 177-185

18 陶文铨. 数值传热学(第 2 版). 西安: 西安交通大学出版社, 2001

19 郭宽良, 孔祥谦，陈善年. 计算传热学. 合肥：中国科学技术大学出版社, 1988

20 李人宪. 有限体积法基础. 北京: 国防工业出版社, 2005

21 张明义, 李双洋, 高志华, 等. 青藏铁路抛石护坡和保温材料复合路基温度场特征非线性分析. 冰川冻土, 2007, 29(2): 306一 314 